\title{
Joint Meeting of the Australian Society for the Study of Brain Impairment and the International Neuropsychological Society, 7-10 July, 2004, Brisbane
}

\section{The Kevin Walsh Encouragement Award for Honours or Masters Research was awarded to Vicki Richards for the following presentation. \\ The Role of Specific Cognitive Impairment and Drug Use in the Development of Post-Operative Delirium in Older Community-Dwelling Adults}

\author{
Vicki A. Richards, ${ }^{1}$ Wayne R. Levick, ${ }^{2,1}$ Wayne G. Reid ${ }^{3}$ and Kichu Nair ${ }^{4}$ \\ ${ }^{1}$ Department of Behavioural Sciences, University of Newcastle, Australia \\ 2 Department of Neuropsychology, John Hunter Children's Hospital, Newcastle, Australia \\ ${ }^{3}$ Department of Neurology, Westmead Hospital, Sydney, Australia \\ ${ }^{4}$ Department of Geriatric Medicine, John Hunter Hospital, Newcastle, Australia
}

Doint prevalence studies indicate that delirium occurs in up to $51 \%$ of surgical and medical inpatients over 50 years of age. Global cognitive impairment and medication use have been identified as risk factors in some studies. No studies have used a range of neuropsychological tests and examined specific cognitive deficits as risk factors preoperatively, in the population of nondemented older adults. This study examined neuropsychological indices and mediation/drug use as predictors of the occurrence of postsurgery confusion/delirium. It was predicted that specific cognitive deficits and medication use may predispose to development of postoperative delirium in older community-dwelling adults. One hundred patients 65 years and over, awaiting elective orthopaedic surgery, underwent a neuropsychological evaluation in the 21 days prior to surgery. The development of postoperative confusion was monitored by reference to patient medical records and by nursing staff using a modified version of the Confusion Assessment method. Results for the 100 participants indicate a $25 \%$ rate of postoperative confusion. For this group, differences between the patients with and without delirium were found on variables of speed of information processing and regular alcohol consumption in the final model. The implications of these findings for the management of this patient population will be discussed. 
The Luria Award for Doctoral Research was awarded to Anthony Angwin for the following presentation.

\section{Comprehension of Syntactically Complex Sentences in Parkinson's Disease}

Anthony J. Angwin, ${ }^{1}$ Helen J. Chenery, ${ }^{1}$ David A. Copland, ${ }^{1}$ Bruce E. Murdoch, ${ }^{1}$ Elizabeth A. Cardell, ${ }^{1}$ John C. Ingram ${ }^{2}$ and Peter A. Stilburn ${ }^{3}$

${ }^{1}$ Centre for Research in Language Processing and Linguistics, Department of Speech Pathology, The University of Queensland, Australia

2 Department of English, Media Studies and Art History, The University of Queensland, Australia

${ }^{3}$ Princess Alexandria Hospital, Brisbane, Australia

R esearch has illustrated that object-relative (OR) sentences (e.g., The girl Rthat the boy pushed bought the food) can be more difficult to process for Parkinson's Disease (PD) patients than subject-relative (SR) sentences (e.g., The girl that pushed the boy bought the food). The role of working memory in sentence comprehension was assessed in a group of 12 participants with PD $($ Age $=63.50, S D=4.70$; Education $=12.08, S D=3.03)$ and 12 healthy control participants matched to the PD group for sex, age and education $($ Age $=61.58, S D=5.16$; Education $=13.33, S D=5.16)$, using a word-by-word self-paced reading task. The task consisted of both SR and OR sentences, with comprehension probes for each sentence. Reading times were recorded for both the noncritical and critical processing regions (critical regions defined as the time taken to read the last word of the embedded clause and the following verb). Accuracy to comprehension probes formed a second dependent variable. Results indicated that while the control group demonstrated significantly slower reading times at the critical processing regions of the OR sentences compared to the SR sentences, the PD group did not demonstrate this difference between sentence types. In addition, while both groups responded to the OR comprehension probes less accurately than the SR comprehension probes during the reading task, only the PD group demonstrated significantly poorer comprehension of OR sentences during an auditory comprehension task. Alternative accounts for the sentence comprehension difficulties evident in PD patients will be discussed, including difficulties experiences by patients with PD in the reactivation of moved syntactic elements.

\section{The ASSBI Travelling Award was awarded to Christine Ernest \\ for the following presentation.}

\section{The Study of Emotional Reactivity Following Unilateral Temporal Lobectomy}

Christine S. Ernest, ${ }^{1}$ David G. Andrewes, ${ }^{1}$ Nicholas B. Allen, ${ }^{1}$ Christine Kilpatrick, ${ }^{2}$ Michael Murphy ${ }^{3}$ and Mark Cook ${ }^{4}$

1 Department of Psychology, University of Melbourne, Australia

2 Department of Neurology, Royal Melbourne Hospital, Parkville, Australia

${ }^{3}$ Department of Neurosurgery, St Vincent's Hospital, Fitzroy, Australia

${ }^{4}$ Department of Neurosciences, St Vincent's Hospital, Fitzroy, Australia

This study examines the contribution of the left versus right anterior tem-
poral lobes to emotional reactivity. Emotional reactions of 21 left-tem-
poral lobectomy patients (LTL), 20 right-temporal lobectomy patients
(RTL) and 20 normal controls (NC) were measured in response to positive,
negative and neutral mood induction conditions using standardised film
clips. Emotional reactivity following each mood induction condition was
assessed using patient self-report, spontaneous facial expressions and heart 
rate responses. Also, subjects were asked to rate the expected reactivity of others for each condition to assess insight into their own reactions. In comparison to NC, the LTL group reported significantly lower negative reactions and higher positive reactions in response to negative stimuli. The ratings of expected reactivity on negative conditions were similar, with the ratings of the LTL group being less negative and more positive than NC. Additionally, LTL patients displayed smaller heart rate changes than NC in response to the fear condition, with a similar trend being observed on all negative conditions. However, on ratings of facial expression, the RTL group displayed significantly less negative facial expressions, specifically fearful expressions, than LTL, while viewing fearful stimuli. In conclusion, LTL patients exhibit lowered emotional responses to negative stimuli and are unaware that their reactions are discrepant. In the presence of a negative emotional elicitor, left-anterior temporal lobe appears to mediate the experiential aspects of emotion, while the right is dominant for spontaneous emotional facial expression, particularly in response to fearful stimuli.

The INS Butters Award was awarded to Melissa Green for the following presentation.

\section{Context Processing and Social Cognition in Schizophrenia}

Melissa J. Green

Macquarie Centre for Cognitive Science, Macquarie University, Australia

Schizophrenia patients show reduced context processing on nonsocial $\checkmark$ cognitive tasks, and poor social cognition on face perception and theoryof-mind tasks. This paper reports two experiments designed to examine social context processing in schizophrenia, when judging the meaning of facial expressions. In the first study, social contextual information was provided in short vignettes that were read to participants immediately before they judged the meaning of a facial expression. Story-face pairs were designed to be discrepant in affective valence so that mental-state judgments reflected the dominance of either situational context or visual information in the faces. Control subjects were influenced by story-contextual information such that faces depicting basic emotions were interpreted according to the situation described in vignettes. Schizophrenia patients showed reduced context processing when the situation suggested a complex mental state (e.g., perceived an angry face as 'anger' even when the context cued 'confusion'), but showed normal use of context when vignettes cued a basic emotional state. A second task examined the processing of visually presented contextual information during a mental state attribution task; visual scanpaths provided a measure of attention to contextual information while participants judged the meaning of facial expressions depicted within social scenes. Paired colour photographs depicting target faces presented in isolation (Series 1) or in the context of a social situation (Series 2), were viewed by participants for 10 seconds each. Group differences in attention to social contextual information were analysed in terms of the number, location, and duration of fixations upon salient features of the scene (faces, objects). Results of both studies support aberrant social-context processing in schizophrenia, and are discussed with regard to previous studies of context processing which have hitherto focused on nonsocial cognition. 
The INS Cermack Award was awarded to Ashok Jansari for the following presentation.

\title{
When Long-Term Memory Does Not Necessarily Mean 'Forever': Evidence of Long-Term Amnesia in a Patient with Temporal Lobe Epilepsy
}

\author{
Ashok Jansari, ${ }^{1}$ Kavus Davis, ${ }^{1}$ Stephanie Firminger ${ }^{1}$ and Narinder Kapur ${ }^{2}$ \\ ${ }^{1}$ School of Psychology, University of East London, London, United Kingdom \\ ${ }^{2}$ Addenbrooke's Hospital, Cambridge, United Kingdom
}

Whereas classical amnesics display an inability to transfer memories to W a long-term store, a new subtype of patients (usually exhibiting epilepsy) are being reported who are able to do this but later present with an inability to retain the information in long-term memory (e.g., Kapur, 1996; Mayes et al., 2003); a phenomenon known as long-term amnesia (LTA). A detailed case study of an LTA patient with mild temporal lobe epilepsy is reported on a range of tasks looking at retention of single words, faces, the Rey Complex Figure and novel stories at 6 different time intervals (over a 4-week period), as well as performance on a range of autobiographical and flashbulb memory tasks. The results indicate a selective deficit in remembering autobiographical memory and event-related memory for information that has not been repeatedly recalled. Subsequent testing looked at the patient's memory for music and investigated the subjective quality of his intact recognition responses using the Recognition of Conscious Awareness (RCA) paradigm. The implications of the findings are discussed in relation to existing theories of long-term memory consolidation.

The INS Rennick Award was awarded to Michelle Roger for the following presentation.

\section{Hemispheric Asymmetry in Olfactory Identification in Mild Cognitive Impairment}

Michelle Roger, Stephen Robinson and Greg Savage

Department of Psychology, Monash University, Australia

$\mathrm{O}$ lfactory dysfunction is a recognised functional impairment associated with the development of Alzheimer's disease (AD). In the last decade, research has begun to explore the possibility of olfactory dysfunction as a marker of the prodromal phase of AD. Research has focused on patients identified as having an increased risk of developing $\mathrm{AD}$ such as those with Mild Cognitive Impairment (MCI). While these studies are suggestive of a higher level of olfactory dysfunction in this population compared with matched controls, not all patients with identified olfactory dysfunction have gone on to develop AD. The majority of these studies have used conventional olfactory identification tests, presenting odours birhinally with a multiple-choice format. This approach fails to take into account the potential presence of unirhinal differences due to underlying asymmetrical neurological changes associated with hemispheric bias to specific pathological processes. Indeed, recent neuroimaging studies suggest hemispheric asymmetry in the pathophysiology of MCI, which affects cortical areas associated with both memory formation and olfactory processing. With olfaction the only human sense which is primarily processed ipsilaterally, it has the potential to be used as a marker of lateralised hemispheric dysfunction in MCI. This paper examines 
the lateralised effects of olfactory function (detection, spontaneous identification and forced-choice identification) in 18 patients with MCI utilising a modified olfactory function assessment with unirhinal presentation. Findings are correlated with cognitive performance on a standardised neuropsychological test battery, and compared with the performance of 26 demographically matched controls. Identification data indicate a left hemisphere bias in impairment, consistent with reports of unilateral onset of $\mathrm{AD}$ pathology. 